Voix et Images

\title{
Denis Vanier, un monstre dans la ruelle
}

\section{Simon Harel et Jonathan Lamy}

Volume 32, numéro 1 (94), automne 2006

Denis Vanier

URI : https://id.erudit.org/iderudit/014700ar

DOI : https://doi.org/10.7202/014700ar

Aller au sommaire du numéro

Éditeur(s)

Université du Québec à Montréal

ISSN

0318-9201 (imprimé)

1705-933X (numérique)

Découvrir la revue

Citer cet article

Harel, S. \& Lamy, J. (2006). Denis Vanier, un monstre dans la ruelle. Voix et Images, 32(1), 8-18. https://doi.org/10.7202/014700ar d'utilisation que vous pouvez consulter en ligne.

https://apropos.erudit.org/fr/usagers/politique-dutilisation/ 


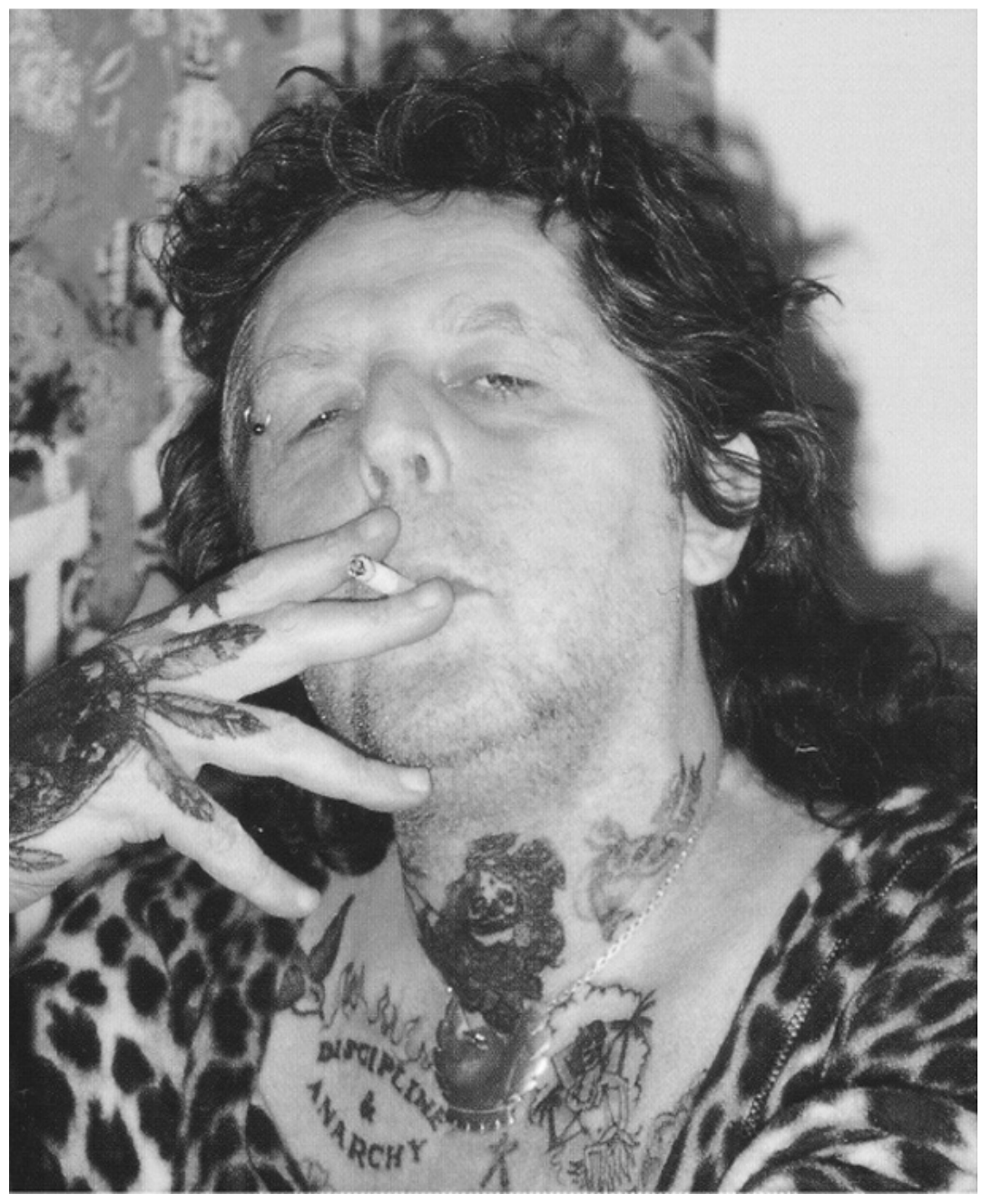

Photographie de Christiane Tremblay

qui figure en couverture du livre Le baptême de Judas. 


\title{
DENIS VANIER, UN MONSTRE DANS LA RUELLE
}

\author{
$+++$ \\ SIMON HAREL \\ Université du Québec à Montréal \\ JONATHAN LAMY \\ Université du Québec à Montréal
}

En dépit de la reconnaissance dont jouit l'œuvre de Denis Vanier (1949-2000), les nombreux discours qui l'entourent - qui se retrouvent dans des journaux, des périodiques, et dans les livres de Vanier, sous forme de préfaces ou de postfaces n'atteignent que rarement le statut d'une véritable étude. Il est désarmant de constater à quel point ce qui a été écrit sur cette œuvre d'une rare intégrité fait surtout référence au personnage qui la chapeaute et qui y fait écran. Vanier est tour à tour un poète important, influent, maudit, tatoué, catholique, terroriste ou junkie, alors que ses textes, eux, ne sont qu'à peine abordés, jamais vraiment analysés ${ }^{1}$. Les livres de Vanier - peut-être en raison de la violence, voire de l'horreur qui les traverse trouvent pour écho des commentaires qui pratiquent le détournement, utilisent des stratégies pour les éviter. Comme l'écrit avec justesse Marcel Olscamp, «[o]n lit toujours Denis Vanier par effraction; il fréquente des lieux tragiques et violents que le lecteur moyen préfère habituellement ignorer ${ }^{2} »$.

Denis Vanier est lu, son œuvre est connue, mais elle n'est pas étudiée. Le présent dossier souhaite combler ce que l'on peut considérer comme une lacune des études sur la poésie québécoise. Nous avons voulu réunir des articles qui, bien que personnels et créatifs, se démarquent du registre du témoignage, dans lequel versent souvent les commentaires sur l'œuvre de Vanier. Dans ce dossier, Catherine Mavrikakis aborde la tension entre le reniement et l'exposition, de même que les images de soi et du corps présentes sur les livres et dans les poèmes de Vanier. Denise Brassard poursuit l'étude du corps à l'œuvre chez Vanier en utilisant les figures du portrait et de l'autoportrait, de la mutation et de l'excarnation. Pour sa part, Thierry Bissonnette mène la double investigation de «l'entrechoquement du désir d'indépendance et de celui d'approbation» et du mythe de la Pentecôte chez l'auteur du Baptême de Judas. Jacques Paquin analyse quant à lui l'utilisation du discours

1 Par exemple, Jean-Guy Pilon, rendant compte de Pornographic delicatessen, écrit dans Le Devoir du 8 mars 1969, p. 21: "Quant aux poèmes eux-mêmes, je ne me sens vraiment pas en mesure d'en dire quoi que ce soit. Cela m'est étranger.» 2 Marcel Olscamp, «La poésie interdite», La poésie au Québec (revue critique 1990), Trois-Rivières, Écrits des Forges, 1991, p. 136. 
médical dans l'œuvre poétique de Vanier, ainsi que la cruauté qui l'accompagne. Finalement, Paul Chamberland s'interroge sur ces motifs de l'excès que sont le meurtre et la traitrise au sein du travail d'écriture de Vanier. Parmi ces réflexions, deux motifs reviennent: les références au catholicisme et la dimension religieuse des poèmes de Vanier, ainsi que la tension entre, d'une part, un désir de singularité, de solitude et, d'autre part, un certain besoin de reconnaissance, de légitimation, auquel participent les nombreuses préfaces des livres de Vanier.

\section{LES RUELLES DE LA POÉSIE}

L'œuvre de Denis Vanier, de même que sa place dans la poésie québécoise, est à l'image de la murale qui lui rend hommage, dans une ruelle près des rues Ontario et Panet (voir figure 1). On en connaît l'existence, on l'évoque parfois, mais on préfère ne pas l'investir de trop près. Le nom de Vanier n'est pas associé à un buste, une place, une école ou une maison de la culture, comme c'est le cas pour Émile Nelligan, Gérald Godin, Anne Hébert ou Marie Uguay, mais à une discrète ruelle du quartier montréalais Hochelaga-Maisonneuve. Dans un poème intitulé "Futur noir», Vanier décrit un contexte géopoétique très sombre:

elle me traîna dans la cour de la douleur

une cour de corps déchirés

de seringues et baisers

de bijoux, cicatrices et tatouages ${ }^{3}$.

La poésie québécoise a très peu traité des lieux que convoque Vanier: bars crasseux, restaurants minables, pizzerias, dépanneurs, pharmacies, buanderies. Autant d'espaces, réels et imaginaires, qui n'ont rien à voir avec l'acceptation habituelle du «poétique» et de ce qui l'incarnerait.

La position particulière de Denis Vanier a également pour cause le fait qu'il n’appartient à aucune génération, courant ou école de la poésie québécoise. Il a commencé à publier très jeune, en même temps que les poètes de la génération qui, en principe, le précède (comme Michel Beaulieu, Nicole Brossard et Raôul Duguay) et bien avant ceux de sa propre génération, sur laquelle, paradoxalement, il a pu avoir une certaine influence (pensons à Claude Beausoleil, à Lucien Francoeur et à Roger Des Roches). La place et les textes de Vanier dans l'histoire de la littérature québécoise se trouvent ainsi marginalisés. En même temps, son œuvre tisse un vaste réseau de dialogues avec d'autres œuvres littéraires, que ce soit par les citations ou les préfaces. Par ailleurs, l'œuvre de Vanier a souvent été associée, de manière anecdotique, aux écrits de Josée Yvon. Nous avons souhaité prendre nos distances à l'égard de cette figure du couple maudit des lettres québécoises, considérant que

3 Denis Vanier, Porter plainte au criminel, Montréal, Les Herbes rouges, 2001, p. 19. 


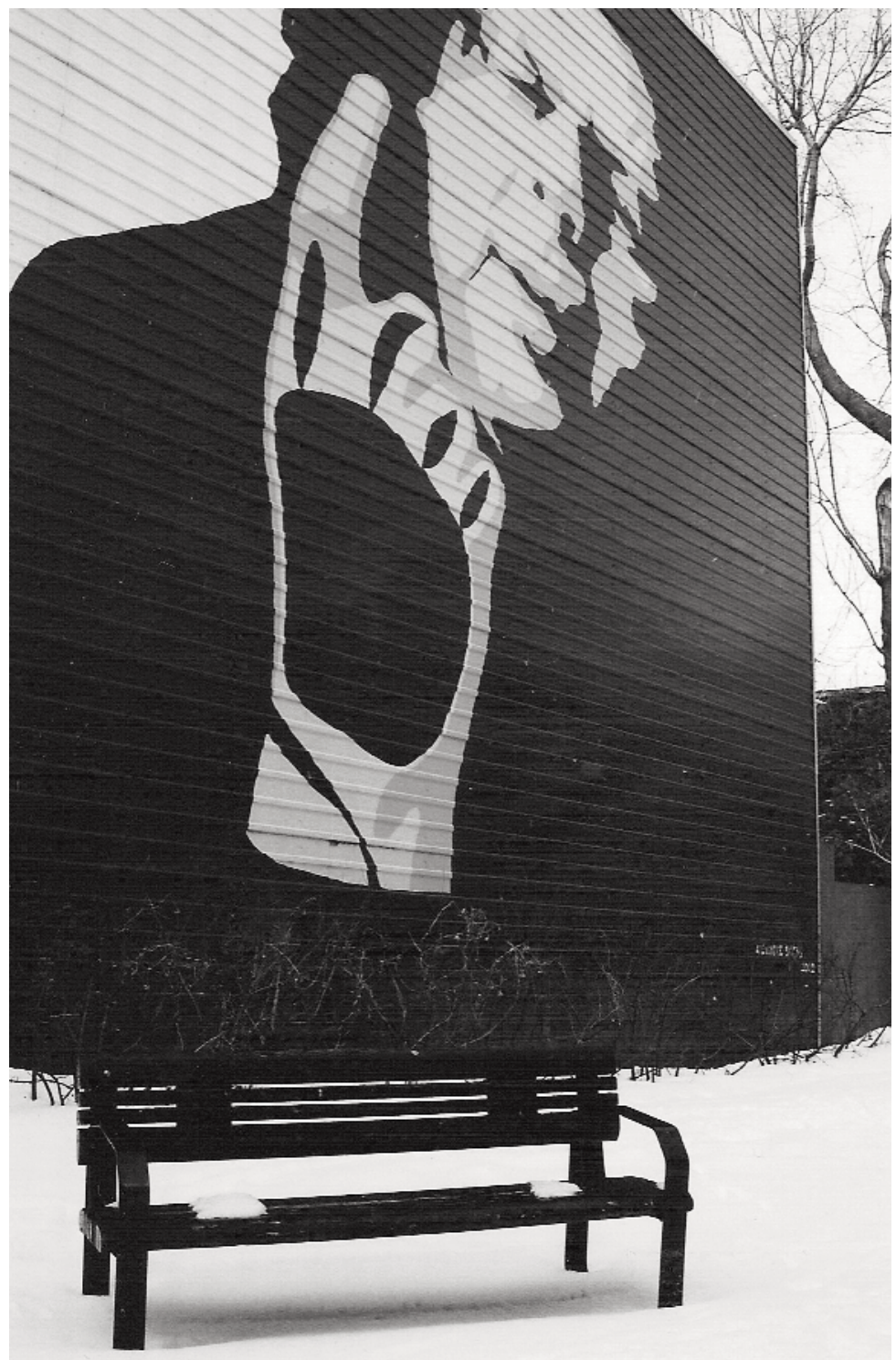

Figure 1. Photographie de Jonathan Lamy, murale "L'ordre du jour» réalisée par Alexandre Bériau d'après une photographie de Victor Virus, Montréal, ruelle Sainte-Rose, entre les rues Panet et Plessis. 
l'écriture de Josée Yvon possède sa propre existence, qu'elle constitue beaucoup plus qu'un complément (son ombre ou son pendant féminin) à la poésie de Vanier.

Les livres de Vanier sont en quelque sorte des ruelles par rapport aux grandes artères de notre poésie. Ils dérangent, agressent et transgressent. L'image de ces "professeurs d'université/égorgés dans le coffre de nos corvettes ${ }^{4}$ ", qu'on retrouve dans Comme la peau d'un rosaire, explique peut-être la quasi-absence de Vanier dans la critique universitaire. Michel Beaulieu pose la question «Qui a peur de Denis Vanier? » et répond en ces termes: "Si la poésie de Denis Vanier nous agresse, c'est qu'elle s'attaque à nos petites vies passives et confortables, c'est qu'elle dénonce la violence qui nous est faite et dont nous ne nous apercevons même plus ${ }^{5}$. " L'œuvre de Vanier met en scène cette "cour de corps déchirés", en montre tous les recoins, y compris ceux qui témoignent de la laideur et de la saleté les plus insoutenables. Dans un des rares textes où il commente son projet poétique, Vanier note:

Il me faut tendre vers l'inénarrable organique, exposer sa chute et ses lents dépôts transparents dans le temps. Outre qu'il soit peut-être inutile et démagogique de le dire, le désespoir est une maladie dont il faut archétyper et symboliser les déchets, que les étapes de cette indisposition soient représentées par images, définitions et traces ${ }^{6}$.

\section{VÉGÉTATION ORDURIÈRE}

Les déchets font partie de la végétation des ruelles. Et l'insoutenable, chez Vanier, est organique. Son œuvre témoigne d'une progression de la pourriture, des «lents dépôts» de ces "déchets racés ${ }^{7}$ ", comme il l'écrit dans Le fond du désir. "Oser en finir », un poème de Porter plainte au criminel, met en scène ce travail de faisandage :

laissez la végétation organique

se nourrir de votre corps

de l'intérieur nous déchargeons ${ }^{8}$.

Il y a dans cette invitation une destruction chimique du corps qui correspondrait à une rétention de l'ordure, une transmutation par laquelle les sujets deviennent des lieux de décharge, où "la seule jouissance dont on se souvienne est celle de/la viande avariée $^{9}$ ». Les corps se posent chez Vanier comme une matière pourrissante,

$$
++
$$

4 Id., Comme la peau d'un rosaire, Montréal, Parti pris, coll. «Paroles», 1977, p. 17. Ces deux vers ont toutefois été retranchés par Vanier lorsqu'il réécrit Comme la peau d'un rosaire pour en faire Sur la route de la soie (voir Euvres poétiques complètes, t. I (1965-1979), Montréal, VLB éditeur/Parti pris, 1980, p. 315). 5 Michel Beaulieu, "Qui a peur de Denis Vanier?», Le Livre d'ici, vol. 6, n 15, janvier 1981, [s. p]. 6 Denis Vanier, «Préface», Une Inca sauvage comme le feu, poèmes biologiques, Québec, Éditions de la Huit, 1992, p. 10, reprise dans Id., L'hôtel brûlé, Trois-Rivières/Moncton/Pantin (France), Écrits des Forges/Éditions Perce-Neige/Le Castor Astral, 1993, p. 8.7 Id., Le fond du désir, Montréal, Les Herbes rouges, 1994, p. 23.8 Id., Porter plainte au criminel, op. cit., p. 82.9 Id., L'hôtel brûlé, op. cit., p. 52. 
à la manière de ce que l'on voit dans certaines œuvres troublantes de la photographe et vidéaste Sam Taylor-Wood ${ }^{10}$.

Les poèmes de Vanier nous disent que les corps et les lieux urbains sont des espaces de détention et d'incarcération. Ses textes sont des formes d'infiltration localisée qui rappellent ces affections qui mettent à mal l'anatomie. Si la poésie de Vanier joue parfois avec le dégueulasse et que la rue Ontario y tient lieu d'enfer dantesque, la localisation de la pauvreté est rendue possible au prix d'un étrange ascétisme. "Chien yang», le premier texte du recueil Le baptême de Judas, débute ainsi :

\author{
Il faut se souvenir \\ du temps où le père \\ portait son sac vert au paradis \\ là d'où provient le mépris ${ }^{11}$.
}

Ce passage est à la fois drôle et sérieux, banal et cruel, dans la mesure où il propose l'image d'une poésie compostée. Le paradis devient un lieu de décharge, où le père (celui de la filiation ou le père divin ?) porte ses déchets qui y pourriront.

Entre l'imaginaire presque «ti-pop» de La petite vie et la crudité de certains poèmes de Vanier, il y a un domaine qu'on n'ose pas vraiment aborder. Bien sûr, l'autodérision est de mise, de même que le désir de saborder la prétention poétique par la mise en scène de l'imagerie prosaïque de l'homme-poubelle, vaine paternité dont le désœuvrement l'apparente à une divinité. Mais il y a plus. Cet univers de l'ordure qui suinte et qui fermente coïncide avec la mise au monde de l'informe. L'ordure, chez Vanier, tient à la fois de l'interjection («Sale ordure!» «T'es rien qu'une ordure!»), d'une matière extrêmement concrète et d'un choix, une sorte de devenir-ordure ou devenir-décharge. C'est une forme de marginalité délibérée, qui consiste à se placer volontairement du côté des poubelles, de la saleté des ruelles, voire à se mettre soi-même à la poubelle, «la grande poubelle qui mène au ciel ${ }^{12}$ ».

\title{
$\bar{A} L^{\prime} A B R I$
}

Dans Hôtel Putama, son seul récit, Vanier formule toutefois le souhait d'ériger un refuge au cœur de la saleté urbaine :

Montréal est une ville sale, imbue de spéculateurs, d'agents doubles, dont aucun terroriste ne daigne même s'occuper. Politiquement, aucun effectif policier n'est plus harassant, dangereux et incompétent qu'en cette ville. L'Est demeure notre

10 Dans le court film Still Life (2001), on assiste, en accéléré, à la décomposition complète d'un bol de fruits. Dans l'œuvre vidéo d'une durée de quatre minutes intitulée A Little Death (2002), il s'agit d'un lièvre. 11 Denis Vanier, Le baptême de Judas, Montréal, Les Herbes rouges, 1998, p. 11.12 Id., Le fond du désir, op. cit., p. 54. 
seul refuge culturel et nous avons strictement l'intention d'y établir un ghetto comme les Mohawks. Noblesse oblige, dans ce cas-ci la mort ne pourra venir que de la part d'un «commis-voyageur» de l'escouade anti-poétique ${ }^{13}$.

Contrairement à un discours multiculturel euphorique - ce que Guillermo GómezPeña nomme le "culti-multuralism ${ }^{14}$ » - , ce ghetto constitue un refuge du même, du semblable, qui s'inspire de stratégies similaires à celles des Black Panthers et des Warriors de la Crise d'Oka de l'été 1990. Toujours dans Hôtel Putama, Vanier écrit ceci à propos de Longueuil :

Elle [Candy, une prostituée] et son amie découvrirent, avec tout l'effroi d'une adolescente de Philadelphie, que les rockers, les punks et les skins furent inventés dans cette petite banlieue de la Rive-Sud bien avant qu'à New York et Los Angeles. Nous étions les White Panthers de la misère et de la poésie ${ }^{15}$.

Dans ce refuge, ce réservoir, cette décharge de bums, il y a une apologie de la délinquance et du terrorisme qu'on ne retrouve que rarement dans la poésie québécoise, et qui consiste à entraver la ville, à la rendre impossible, dysfonctionnelle. En même temps, ce noble ghetto peut être perçu comme un petit pays de cow-boys et d'Indiens, comme dans les films westerns de série B. Il y a là quelque chose de profondément enfantin. Se créer un refuge au sens où les enfants le font, tel qu'on le voit dans le film d'André Melançon La guerre des tuques (1984), constitue un projet naïf, mais qui ne s'oppose pas nécessairement à une clairvoyance qui trouve sa matière dans la dégénérescence urbaine.

Vanier fut avant tout un poète local. L'expression n'est pas péjorative. Au contraire, elle met l'accent sur le caractère circonscrit de ses déambulations. Les ruelles et le "garage de cosmétiques ${ }^{16}$ » deviennent alors un abri, un lieu-fétiche que le poète parcourt sans relâche. Dans cette marche au cœur d'un territoire balisé, le sujet peut alors déclamer, d'une voix sourde, ces maîtres mots que sont l'intégrité et la résistance, qui consistent à ne pas céder sur l'intensité de son désir. L'acte de résistance rappelle, dans un premier temps, les figures un peu vieillottes de la guérilla urbaine. Des White Panthers aux Devil's Disciples, cet imaginaire discordant offre l'image d'un univers de mécréants auquel l'œuvre de Vanier est très souvent associée. C'est oublier cependant que la rébellion est aussi un jeu. Dans les évocations de violence et de mafias urbaines, on serait en peine d'identifier une liberté de création. Pourtant, l'œuvre de Vanier est attentive, malgré le tohu-bohu urbain, à un art de la feinte. C'est un peu ce qu'on retrouve dans «Corps de feu»:

$$
+++
$$

13 Id., Hôtel Putama. Textes croisés, Longueuil-New York, 1965-1990, Québec, Éditions de la Huit, 1991, p. 58. 14 "An Esperantic Disney world view in which all cultures, races and sexes live happily together.» Cette définition se trouve dans Guillermo Gómez-Peña, The New World Border, San Francisco, City Lights, 1996, p. 241. 15 Denis Vanier, Hôtel Putama. Textes croisés, Longueuil-New York, 1965-1990, op. cit., p. 33.16 Id., Renier son sang, Montréal, Les Herbes rouges, 1996, p. 67. 
J'ai besoin d'être attendri

comme un steak de léopard

fuir dans les trapèzes,

mordre l'écuyère au talon,

étouffé sous le mensonge de la couleur ${ }^{17}$.

On sera peut-être surpris de remarquer la présence d'un imaginaire du cirque dans les écrits de Vanier. Qu'on ne se méprenne pas quant à la teneur de la référence. Nul Cirque du Soleil, aucune représentation normale de la festivité ou des arts de la rue. Le ghetto chez Vanier n'est pas un thêâtre où l'étiquette et les convenances sont de mise. L'imagerie du cirque est au contraire ramenée à sa plus simple expression. Parfois, de vraies bêtes mangent le dompteur. Quant au funambule, il perd pied et s'écrase sur la piste. Dans l'évocation de ce "cirque de chair ${ }^{18}$ ", pour reprendre une image de Je, les poèmes de Vanier ont un petit côté gore, témoignent d'une forme d'horreur où l'humour se manifeste fréquemment. L'espace du cirque et de l'ordure participe d'une destruction du soi. Le dernier poème du recueil Le baptême de Judas, «Donner la faim", met en scène ce saccage: "Que serait ce miroir/sans steak haché pour l'alourdir ${ }^{19}$.»

\section{UNE CACHE DE MONSTRES}

Si la végétation des ruelles est composée d'ordures, sa faune est peuplée de monstres, comme ceux que l'on voit dans le documentaire de Charles Binamé intitulé Denis Vanier présente son show de monstre ${ }^{20}$. Dans ce film, qui met en scène beuveries, bagarres, vols à l'étalage et consommation de drogues, il y a, comme dans les poèmes de Vanier, une oscillation entre le désir de (se) cacher et celui de (se) montrer, qui rejoint le paradoxe du monstre, de la bête de foire, qu'on exhibe et qu'on voile tour à tour. Le monstre nous dit: regardez les tatouages, les cicatrices, regardez les ordures, la viande faisandée. Mais, à l'autre versant de cette valsehésitation, il se terre, à l'abri des regards, fracasse le miroir, le fait disparaître dans un tumulus de viande hachée.

Le refuge urbain est à la fois une cache (semblable à toutes ces cachettes que les enfants-adultes vaniériens recherchent au cœur des ruelles) et un theâtre en plein air, un freak show. Dans cette singulière dynamique, qui rappelle le cabinet des curiosités, la délinquance urbaine, le recel, la vente de drogues et la prostitution sont les passages obligés d'un cirque ordinaire. Se cacher, se faire tout petit alors qu'on se sait traqué, n'est-ce pas recourir à l'idée de tactiques déconcertantes, paradoxales? Denis Vanier présente son show de monstre et plusieurs poèmes de Vanier

17 Id., Le baptême de Judas, op. cit., p. 14.18 Id., Euvres poétiques complètes, t. I (1965-1979), op. cit., p. 62. 19 Id., Le baptême de Judas, op. cit., p. 68. 20 Charles Binamé, Denis Vanier présente son show de monstre, Montréal, Radio-Québec, série «La vie qu'on mène», 1974, 60 min. Scénario de Denis Vanier et Josée Yvon. 
donnent l'impression d'un décor de carton-pâte, où se déroule un jeu de cache-cache hardcore. Dans cette alliance d'une culture trash et d'une poésie urbaine cantonnée à un repère de monstres, il y a hors de tout doute un discours neuf qui rompt avec les formes trop polies de l'inconvenance et de l'irrévérence.

La forme composite de l'œuvre de Vanier est proprement monstrueuse. Bandes dessinées, collages de journaux à potins alternent avec des illustrations de gravures de William Blake ou inspirées de Dante. Cette stratégie, qui s'apparente à bien des égards à la figure du recel, ne se limite pas aux formes diverses de la culture lettrée et, de manière plus générale, de l'univers scripturaire. Les écrits de Vanier font cohabiter la représentation photographique de tatouages, d'accessoires de sexshops et des exergues du comte de Lautréamont, du marquis de Sade, de Friedrich Hölderlin. Dans tous les cas, il s'agit de promouvoir un mouvement de contrebande qui justifie l'apparent éclectisme cher à Vanier. Certains diront peut-être qu'il y a peu de similitudes entre, par exemple, la reproduction d'une seringue hypodermique et l'iconographie religieuse. Pourtant, c'est là commettre une grossière erreur dans la mesure où les écrits poétiques de Vanier mettent en valeur cette hétérogénéité impure de sources citationnelles apparemment hétéroclites.

Des commentateurs, ainsi Lucien Francoeur, notamment dans la préface à Lesbiennes d'acid ${ }^{21}$, ont fait de Vanier un roi dans son royaume-ghetto. Cet éloge est simpliste tant il renoue avec l'imagerie surannée de l'archange rocker des années 1960. Si nous lisons avec attention l'œuvre de Vanier, nous y trouvons la fragile description d'un maître de cérémonie qui présente son univers hybride aux badauds de l'Est de Montréal ou de Longueuil. Dans un poème faisant partie du recueil Renier son sang, "Cette pieuvre pastel crachée au visage», Vanier écrit:

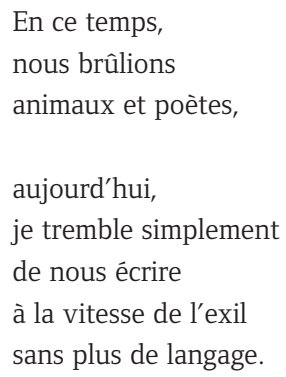

Rien de tendre

toujours le livre:

l'histoire de chevaux de manèges

et d'un tireur de couteaux ${ }^{22}$. 
Cette description décape l'image du cirque ordinaire. Le constat de vieillissement coïncide ici avec un aveu de monstruosité terrible, comme le sont les derniers recueils de Vanier, d'une dureté de plus en plus implacable. Après s'être fait le magesorcier d'un "show de monstre", tireur de coutelas sur des cibles inventées, poseur de bombes et de pièges, Vanier se place sous la lunette des francs-tireurs et risque sa peau. La rue Ontario n'est plus alors la représentation d'un paysage qui offre asile aux marginaux, aux désespérés et intoxiqués de l'Est de Montréal. Le sujet poétique se fond dans un univers où la pollution, l'abjection sont les seuls recours. Ce retournement de la violence sur soi consacre l'immobilité du poète; une bien cruelle ascèse prend forme. Ressemblant à un stylite, juché au sommet d'une colonne dans l'exercice de la prière, Vanier, poète-ordure-bouddha, dicte ses poèmes, immobile comme un feu de signalisation à l'intersection des rues Ontario et Panet.

\section{LA SOLITUDE D'UN CONTAINER}

En lieu et place d'un tombeau littéraire, cet espace dédié aux grands hommes, le poète Vanier hante une ruelle montréalaise, habite un conteneur de déchets ou de viande avariée. Après avoir établi son repaire dans l'Est montréalais, le bunker devient un espace carcéral et cancéreux. À lire Vanier, l'espace urbain n'est plus la plaque tectonique de nos imaginaires migrants. $\mathrm{Si}$, depuis la décennie 1980, les expressions de déterritorialisation et d'altermondialisme, comme antidotes au néolibéralisme, ont été popularisées, Vanier est demeuré un poète solitaire. Dans son œuvre, Montréal devient une ville-ghetto, un univers dont la signalétique urbaine est faite de matériaux synthétiques sans noblesse apparente. Banquettes de vinyle dans des bars et brasseries décatis, plastiques usés aux couleurs criardes, le règne du cheap et du design rudimentaire (du 5-10-15 au Dollarama de la misère ordinaire) colle à la peau.

Si Denis Vanier peut être considéré aujourd'hui comme un poète de la rue, ce n'est pas, comme on l'imagine trop souvent, parce qu'il aura été un marginal, un drogué, un franc-tireur. Ces expressions ont l'heur de plaire tant elles décrivent une méchanceté littéraire de peu d'envergure, une soif de provocation qui recourt aux poncifs les plus éculés de la dissidence, de la contestation et, pourquoi pas, de l'altercontestation. Vanier adopte un autre point de vue dans son écriture poétique, privilégiant le recel, la contrefaçon: figures peu connues, sous-estimées, de son arsenal poétique. En effet, il serait trop facile de réduire l'œuvre de Vanier aux formes établies de l'hybridité culturelle. Nous avons indiqué un peu plus tôt que l'écriture de Vanier était locale tant elle fait de l'Est de Montréal son fonds de commerce. L'expression semblera à certains déplacée, à tout le moins dévalorisante. Mais ce fonds de commerce n'était-il pas, pour lui, une façon de promouvoir une poésie de contrebande, un véritable art de la rue qui rejette du revers de la main toute comptabilité littéraire au profit d'un recel autrement plus inquiétant?

À l'encontre du provincialisme qui hante le discours littéraire québécois et qui revendique le droit à l'universalité, Vanier, tel un corsaire, le tranchant de la lame de couteau entre les dents, débarque en ville. Que faire de cet énergumène, ce «Juif 
mohawk » tatoué qui transforme Montréal en freak show, alors même que cette ville tente de gagner ses lettres de noblesse dans le club restreint des villes festives et créatives? L'œuvre de Vanier ne se contente pas, on s'en doute bien, de reprendre à son compte l'éloge de la dérive urbaine. À vrai dire, Vanier est un ambassadeur de la désolation, qui nous oblige à tenir compte des violences et des impostures qui entachent la perception fortement idéalisée d'identités culturelles subversives et marginales.

Vanier accepte d'être cet habitacle de la misère humaine, la pièce rapportée d'un monde industriel anonyme. On sait que les passeurs de drogue et de biens de contrebande font un usage répété de ces containers (ou conteneurs) que l'on retrouve à proximité des gares ferroviaires et des ports. L'agencement géométrique de ces sculptures monumentales, nous offre, encore une fois, l'image d'un monde en proie au recel. Qu'il s'agisse de réfugiés qui s'enferment dans ces matrices métalliques pour mieux être transportés d'un continent à l'autre, du trafic de biens que l'on fait circuler dans l'illégalité, le container, forme froide, muette, aux angles acérés, renoue avec les images les plus dures de l'histoire contemporaine. Sans qu'il soit question ici de mettre en relation, ce qui serait d'une absolue maladresse, la réalité crue des déportations, rapts et disparitions avec l'œuvre poétique de Vanier, il convient néanmoins de prendre au sérieux cette image du container, véritable lieu de mort sis au cœur de l'Est montréalais. Chez Vanier, la poubelle, le cercueil métallique, le bunker tiennent lieu à la fois de fosse commune et de paradis. Dans un poème de L'hôtel brûlé, on peut lire: «J'ai trop vécu dans la fosse commune/pour même mort m'en ennuyer ${ }^{23}$.» Ailleurs, Vanier écrit:

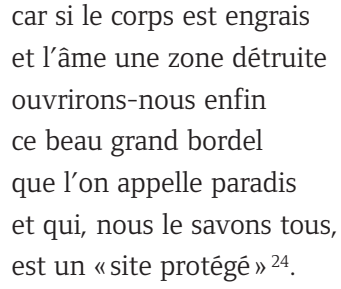

Avec les déchets, ce sont les cadavres qui emplissent et engraissent la décharge, le repère, le ghetto. Les corps, les ordures et les monstres finissent dans le même bordel, la même ruelle.

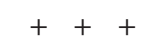

23 Id., L'hôtel brûlé, op. cit., p. 47.24 Ibid., p. 31. 